\title{
CARACTERÍSTICAS SENSORIAIS DE COMPOTAS DE PÊSSEGO LIGHT ELABORADAS COM SUCRALOSE E ACESULFAME-K
}

\author{
Carla R. B. MENDONÇA', Rui C. ZAMBIAZI ", Márcia A. GULARTE , Graziele G. GRANADA
}

\section{RESUMO}

O trabalho objetivou realizar uma caracterização sensorial de compotas de pêssego com reduzidas calorias. As compotas foram elaboradas pela substituição parcial de açúcar pelos edulcorantes sucralose, acesulfame-K e sucralose+acesulfame-K. Além desta caracterização, investigou-se a vida-de-prateleira destes produtos durante um período de 90 dias. Para isto, foram avaliados os atributos de aparência geral, cor, brilho, maciez, sabor geral e acidez, logo após o processamento, aos 30, 60 e 90 dias de estocagem. Ao término do periodo de estocagem executou-se, ainda, um teste de preferência. De acordo com a avaliação sensorial dos diferentes atributos, detectou-se diferenças significativas entre as quatro formulações de compota, especialmente em relação aos atributos de sabor e acidez. As formulações com acesulfame-K e sucralose+acesulfame-K mostraram valores inferiores às demais. Durante o periodo de armazenamento não se constatou expressivas alterações nas características sensorias das compotas. Os resultados do teste de preferência mostraram que não houve distinção na preferência dos consumidores por qualquer das formulações.

Palavras-chave: compota light; análise sensorial; sucralose; acesulfame-K; pêssego.

\section{SUMMARY}

SENSORY CHARACTERISTICS OF LIGHT PEACH COMPOTE ADJUSTED WITH SUCRALOSE AND ACESULFAME-K. The objective of this work was carried out as a sensory characterization of low calorie peach compote. The products were adjusted with the partial substitution of sugar by sucralose, acesulfame-K and sucralose+acesulfame-K. The shelf life of these products during a period of 90 days was also evaluated. The attributes of appearance, colour, shiny, softness, flavour and acidity were also evaluated soon after processing and after 30,60 and 90 days of storage. A preference test at the end of the storage period was also performed. According to the sensory evaluation of the attributes significant differences were detected among the four formulations of peach compote, especially in relation to the attributes of flavour and acidity. The formulations with acesulfame- $\mathrm{K}$ and sucralose+acesulfame-K showed lower values than the others. During the storage period expressive changes in the compote sensory characteristics were not observed. The results of the preference test showed that there was no difference in the consumers preference for any of the formulations.

Keywords: light peach compote; sensory analysis; sucralose; acesulfame-K.

\section{1 - INTRODUÇÃO}

A história de uso de açúcar provém da afinidade dos seres humanos por substâncias doces; estudos fisiológicos e psicológicos ressaltam a importância do uso de substâncias doces em alimentos e bebidas, pois a doçura pode ser percebida até mesmo pelo feto. Desde as primeiras horas de vida, a sensação de doçura produz expressões faciais que podem ser interpretadas como indicativo de prazer [3, 8]. Os açúcares funcionam como combustíveis do metabolismo humano, e suas características sensoriais são indicativas desta capacidade [1].

\footnotetext{
Recebido para publicação em 11/05/2001. Aceito para publicação em 31/08/2005 (000645).

Departamento de Química Orgânica, Universidade Federal de Pelotas.

Departamento de Ciência dos Alimentos, Universidade Federal de Pelotas. (UFPel). Endereço: Rua Prof. Araújo, n. 1067, Apto 402 Centro. CEP: 96020-360,Pelotas-RS.E-mail:zambiazi@ufpel.tche.br Departamento de Educação Física e Saúde, Universidade de Santa Cruz do Sul.

A quem a correspondência deve ser enviada.
}

Porém, o incremento do uso de sacarose na dieta recebeu grande atenção nos Estados Unidos em meados dos anos sessenta, época em que alguns efeitos adversos foram observados em certos segmentos da população, como doenças coronárias crônicas, obesidade, diabete e hipertensão, as quais foram atribuídas ao consumo excessivo de açúcar [1]. Desde então, alternativas para substituição da sacarose por outros adoçantes tornaram-se uma imposição e, a pesquisa sobre edulcorantes passou a receber especial atenção [3, 30]. Integrado as novas tendências, vem crescendo o número de edulcorantes de baixo ou nenhum valor calórico, disponiveis para aplicações em alimentos e bebidas, vindo a constituir uma das áreas mais dinâmicas no campo dos aditivos [4, 28, 38]. Os compostos utilizados como adoçantes estimulam a sensação doce por interagirem com os receptores gustativos da língua e garganta [1].

Existem alguns aspectos principais sob os quais os edulcorantes são avaliados, estes incluem a segurança, solubilidade, estabilidade, sabor e custo [13, 14, 28, 31]. A análise sensorial é a principal técnica usada para avaliar o sabor de produtos alimentícios que contêm edulcorantes [23]. Durante a formulação de um alimento de baixas calorias, muitos fatores podem mascarar ou intensificar as características de sabor de um edulcorante e podem gerar diferenças discretas ou pronunciadas; a análise sensorial é a técnica tipicamente aplicada na definição de doçura [32] pois, um dos instrumentos que mede a do- 
çura é a lingua e existe, inevitavelmente, uma variação estatística de percepção entre diferentes indivíduos [37]. No estudo da avaliação de intensidade de doçura é comum comparar as substâncias com soluções de sacarose [13].

Nos últimos anos, a análise sensorial dos alimentos deixou de ser uma atividade secundária e empírica e enquadrou-se na categoria de disciplina científica, capaz de gerar informações precisas e reprodutiveis, sobre as quais recaem importantes decisões, relativas à seleção de matérias-primas, modificações e padronização de métodos e, otimização de formulações para desenvolvimento de produtos; a avaliação sensorial torna-se uma ferramenta básica para aplicação na indústria de alimentos [25]. A crescente incorporação de técnicas de análise estatística e as facilidades para o processamento de dados em software específicos, não só permitem o manejo da informação originada da análise sensorial, como também estão dando lugar a novos métodos sensoriais, capazes de prover informações, até então, não possíveis por qualquer outro método instrumental [26, 35].

O objetivo deste trabalho foi verificar, através de uma análise sensorial, a viabilidade da substituição parcial de açúcares por sucralose na elaboração compotas de pêssego com reduzidas calorias. A fim de estabelecer comparação, efetuou-se também a substituição parcial dos açúcares por acesulfame-K, e pela combinação de sucralose e acesulfame-K. Intencionou-se caracterizar sensorialmente as compotas de pêssego light, bem como avaliar a estabilidade sensorial dos produtos ao longo de 90 dias de estocagem, além de identificar o efeito sinérgico da mistura sucralose e acesulfame-K, e a preferência dos consumidores pelos produtos elaborados.

\section{2 - MATERIAL E MÉTODOS}

\section{1 - Material}

Para elaboração das compotas foram utilizados os edulcorantes sucralose, sob a forma líquida na concentração de 3,1\% (marca comercial Splenda) e acesulfame$\mathrm{K}$, na forma sólida, com $100 \%$ de pureza (doado pela Companhia Hoescht, sob a marca Sunett); adoçantes sacarose e glicose adquiridos no comércio local, e pêssego da espécie Prunus persica (L.) Batsch, cultivar Precocinho (oriundo do interior de Pelotas/RS, safra 1998/1999; disponibilizado por indústria local).

\section{2 - Processo de elaboração das compotas}

As compotas de pêssego foram elaboradas em linha de processamento industrial. Inicialmente o pêssego passou pelas operações de corte e descaroçamento mecânico, após enviado à pelagem (efetuada com hidróxido de sódio a $15 \%$, a $90^{\circ} \mathrm{C} / 1 \mathrm{~min}$ ), seguindo para uma lavagem em jatos de água sob pressão. Após sua classificação, o pêssego foi acondicionado em embalagens de vidro, pesado e adicionado à calda. Foram preparadas quatro formulações de calda, sendo uma controle, elaborada dis- solvendo-se os açúcares sacarose e glicose $(8: 2 \mathrm{p} / \mathrm{p})$ em água, de modo a obter uma calda inicial a $30^{\circ}$ Brix. As outras três caldas foram preparadas pela substituição de parte dos açúcares pela quantidade equivalente em doçura de edulcorantes. Para o cálculo da doçura equivalente, utilizou-se as indicações de CÂNDIDO \& CAMPOS [9] e o limite máximo de edulcorantes em produto alimentício estabelecido pela legislação brasileira [6, 7]. Foram elaboradas caldas a $19 \mathrm{Brix}$, as quais tiveram a doçura complementada em relação ao controle, com sucralose $(0,0189 \%)$, acesulfame-K $(0,0385 \%)$ e com a mistura de sucralose+acesulfame-K na proporção de 1:1 (0,0094\% sucralose e 0,0192\% acesulfame-K). Após a adição da calda, foi realizada exaustão por $3 \mathrm{~min}$., seguindo tratamento térmico por 15 minutos a $100^{\circ} \mathrm{C}$, e resfriamento pela injeção água fria por 15 minutos.

\section{3 - Análises sensoriais}

Realizou-se a avaliação sensorial dos produtos elaborados, logo após o processamento, aos 30, 60 e 90 dias de estocagem. Para o aspecto aparência foram avaliados os atributos cor, brilho e aparência geral; em relação à textura foi avaliada a maciez; avaliou-se também o sabor geral e a acidez das compotas. A escolha destes atributos para avaliação, baseou-se no fato de constituírem os atributos de primeira impressão, que têm grande influência no consumo de produtos alimentícios [27].

Para os testes sensoriais utilizou-se uma equipe de 15 avaliadores treinados, sendo 12 do sexo feminino e 3 do sexo masculino, com idades entre 18 e 50 anos. Inicialmente, foram selecionadas através de uma ficha convite, 18 pessoas para receberem o treinamento, após o mesmo, determinou-se os que participariam das avaliações. Para o treinamento e seleção da equipe seguiu-se as indicações da INTERNATIONAL ORGANIZATION FOR STANDARDIZATION $[19,20]$, realizando-se os testes de gostos primários; limiar absoluto, para gosto doce e acidez, usando soluções de sacarose e ácido cítrico em diferentes concentrações; ordenação; comparação múltipla; escala de categorias; pareado-grau de diferença e avaliação de atributos. Os padrões utilizados nos testes foram: soluções de sacarose, ácido cítrico, sulfato ferroso, glutamato monossódico, cloreto de sódio, cafeína e compotas de pêssego light e convencional de diferentes marcas e procedências. O treinamento foi desenvolvido durante 35 sessões ao longo de três meses, em cabines individualizadas de acordo com a recomendação da ISO [18]. Os 15 avaliadores selecionados foram os que apresentaram melhor memória sensorial, habilidade e reprodutibilidade das respostas, medidos através da tabulação e desvio padrão dos dados obtidos durante o treinamento. Os julgadores analisaram as amostras pelo teste de avaliação de atributos, utilizando-se uma escala de intervalo estruturada de 1 a 9 em que, para os atributos aparência, cor, sabor e acidez o valor 1 referia-se a condição péssima, nada característico à compota de pêssego e o valor 9 a ótimo, totalmente característico a compota de pêssego; para o atributo brilho, o valor 1 referia-se a condição sem 
brilho e o valor 9 a extremamente brilhoso; para o atributo maciez o valor 1 referia-se a condição péssima, considerada como extremamente mole/dura e o valor 9 a ótimo, condição ideal [17]. Para a execução dos testes, as amostras foram apresentadas aos julgadores uma de cada vez, em recipientes pequenos de porcelana branca, codificados com números de três dígitos aleatórios; cada amostra foi constituída de uma metade de pêssego e aproximadamente $20 \mathrm{~mL}$ de calda, a temperatura ambiente [16], seguindo as mesmas condições ambientais do treinamento [18].

Aos noventa dias de armazenamento foi realizado um teste de preferência dos consumidores pelas 4 formulações de compotas através do teste de ordenação. Para este teste utilizou-se 100 consumidores não treinados, aos quais foi solicitado que ordenassem as amostras da mais preferida a menos preferida; tendo-se atribuido o valor 1 a mais preferida e o valor 4 a menos preferida [35]. As amostras foram apresentadas aos consumidores da mesma forma anteriormente citada, no entanto, todas de uma só vez.

\section{4 - Análise estatística}

Todos os resultados foram avaliados por análise de variância (ANOVA). Para efetuar a análise de variância, todos os dados foram previamente transformados para se enquadrar dentro das condições pressupostas para a análise de variância. Os valores que mostraram diferença significativa de médias, foram complementados com o teste de Duncan, ao nivel de $5 \%$ de probabilidade ( $\mathrm{p} \leq 0,05)$, usando o programa Statistica 5.1 [34].

\section{3 - RESULTADOS E DISCUSSÃO}

A Tabela 1 apresenta os resultados da avaliação sensorial das compotas de pêssego, ao longo do período de estocagem.

Durante os meses de armazenamento, a compota de pêssego elaborada com sucralose não apresentou diferenças significantes para os atributos cor, maciez, sabor e acidez; somente para o atributo brilho observou-se uma diferença significativa entre os tempos 30 e 90 dias $(p=0,028)$. Pelos dados de sabor e acidez evidencia-se a estabilidade deste edulcorante [2, 15, 21, 29], verificandose também que sucralose não conferiu sabor estranho às compotas, concordando com a afirmativa de que este edulcorante não confere sabor estranho nos produtos em que é empregado [13, 33, 40].

A compota de pêssego com acesulfame-K, quando avaliada sensorialmente ao longo dos 3 meses de armazenamento, mostrou que os atributos, aparência, maciez, sabor e acidez não apresentaram variações significativas, sendo que houve diferenças significativas entre os tempos 30 e 90 dias com relação a cor $(p=0,049)$ e ao brilho ( $\mathrm{p}=0,038)$; tendo, em ambos os casos, no tempo de

TABELA 1 - Média dos valores obtidos pela avaliação sensorial das compotas do pêssego durante o armazenamento

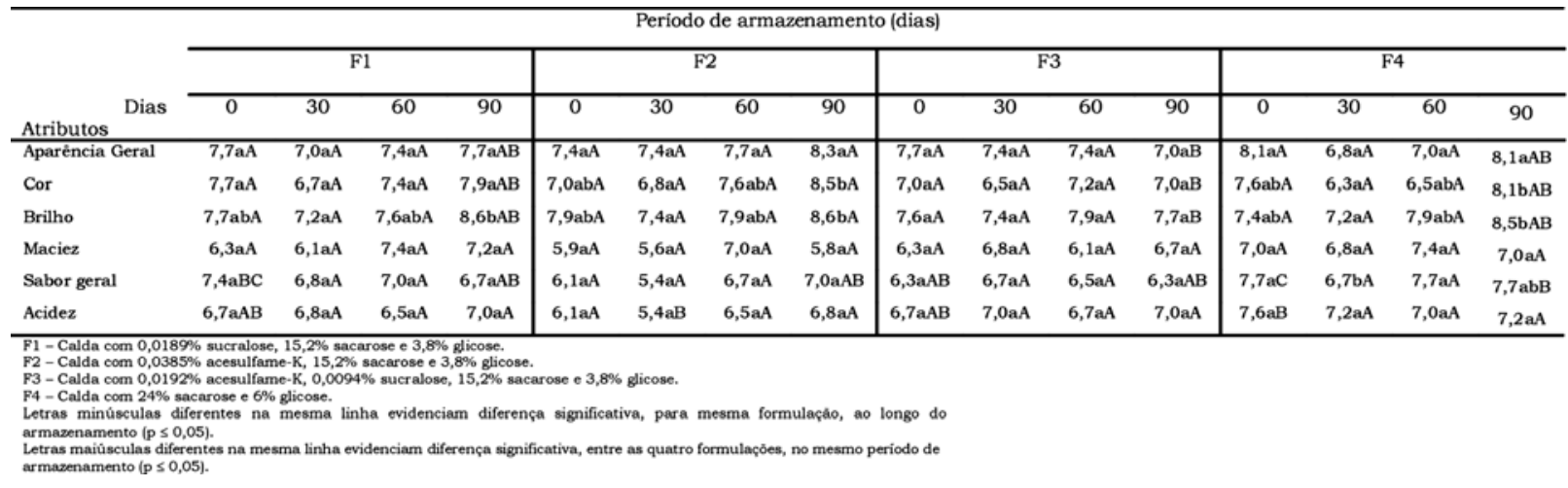

TABELA 2 - Intervalo de confiança dos atributos sensoriais das compotas de pêssego

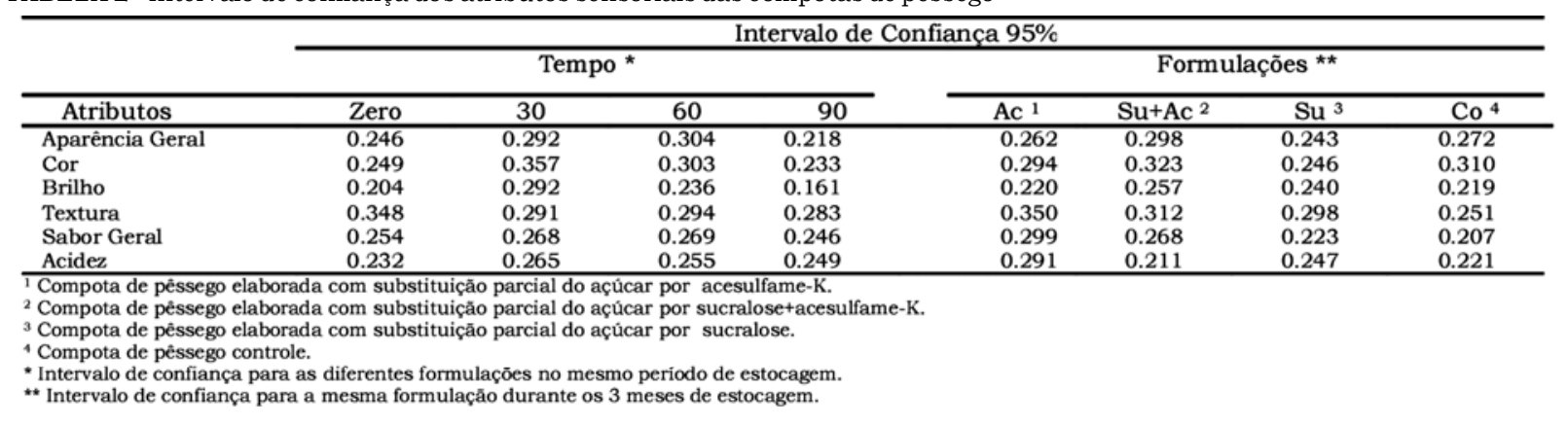


90 dias de armazenamento apresentado maiores valores.

Esses resultados indicam que mesmo após 90 dias de armazenamento não ocorreu decréscimo em suas características sensoriais, sendo que aparência, cor, brilho, sabor e acidez foram até mesmo considerados melhores neste período. $\mathrm{O}$ fato do sabor e acidez manteremse inalterados sensorialmente, deve-se possivelmente à estabilidade do edulcorante acesulfame-K [10, 12, 30], que manteve seu potencial edulcorante na compota de pêssego.

A compota de pêssego elaborada com a combinação dos edulcorantes sucralose+acesulfame-K, apresentou pequenas variações nos resultados das análises sensoriais; no entanto, sem apresentar qualquer efeito significativo entre os dados obtidos ao longo de 3 meses de armazenamento. Assim, o uso combinado dos edulcorantes, em partes equivalentes, demonstrou maior estabilidade, considerando todos os atributos sensoriais avaliados, que o uso individualizado nas compotas de pêssego.

Os diversos tipos de edulcorantes apresentam características diferentes, que podem beneficiar um produto quando combinados [24]; quando a combinação apresenta efeito sinérgico, tem-se uma maximização no sabor e redução no residual estranho [11, 22], no entanto, algumas combinações apresentam melhor performance em alguns produtos do que em outros [12]. Neste estudo, a combinação de sucralose e acesulfame-K apresentou pequena vantagem em relação a acesulfame- $\mathrm{K}$ isolado, nas compotas de pêssego, considerando o atributo sabor. De acordo com SUNETT [36], o sabor é um dos fatores principais que influenciam a decisão de consumo de alimentos processados; assim, o sabor dos produtos com redução de calorias, não pode ser diferenciado dos produtos convencionais.

A interação entre os adoçante é complexa e os resultados variam entre os diferentes sistemas alimentares [39]. BORNSTEIN, WIET \& POMBO [5] sugerem que a mistura de dois edulcorantes pode beneficiar o perfil de doçura, proporcionando maior facilidade de detecção e maior duração da doçura. TORNOUT, PELGROMS \& MEEREN [38], afirmam que misturas de edulcorantes com açúcar têm sido bastante usadas, pois além de mascarar o residual amargo de alguns edulcorantes, conferem "corpo" aos produtos.

De acordo com VERDI \& HOOD [39], misturas de sucralose com acesulfame-K demonstram sinergismo entre 7,2 e $9,8 \%$. Isto significa que, quando os edulcorantes são misturados, guardando a equivalência de doçura de cada um, verifica-se uma elevação na doçura da ordem de 7,2 a $9,8 \%$.

A análise estatística dos resultados obtida para a formulação de controle demonstrou a não existência de diferença significativa ao longo do armazenamento para o os atributos aparência, maciez e acidez; e a existência de diferença significativa entre os tempos 30 e 90 dias quanto a cor $(p=0,048)$ e ao brilho $(p=0,047)$; sendo que aos 90 dias, os resultados obtidos para cor e brilho foram considerados superiores aos obtidos no tempo de 30 dias. Também, foi observada uma diferença significativa entre o tempo 0 e 30 dias $(p=0,044)$, e entre o tempo 30 e 60 dias ( $p=0,047)$ com relação ao sabor; neste caso, aos 30 dias apresentou valores inferiores aos demais.

As Figuras 1, 2, 3 e 4 apresentam os resultados obtidos para o perfil sensorial das quatro formulações de compotas de pêssego, durante o período de armazenamento.

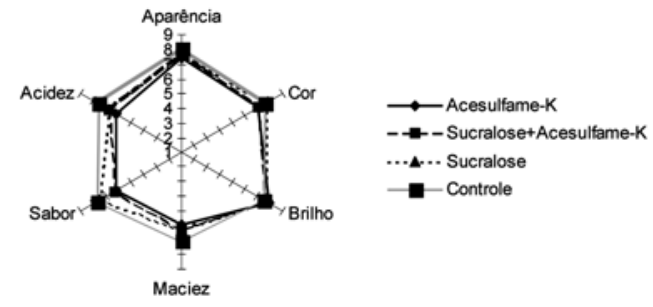

FIGURA 1 - Perfil de características das compotas de pêssego logo após o processamento

Embora tenha ocorrido pequena variabilidade entre os valores médios atribuídos pelo julgadores para os atributos aparência, cor, brilho e maciez, os resultados sensoriais obtidos no tempo zero não evidenciaram diferença significativa entre as quatro formulações, ao nível de $5 \%$ de probabilidade.

Porém, os valores conferidos aos atributos sabor geral e acidez evidenciaram diferenças significativas entre as formulações. As diferenças significativas para o atributo sabor geral ocorreram entre a formulação controle e a formulação elaborada com sucralose+acesulfame-K $(p=0,035)$; entre a formulação controle e a elaborada com acesulfame- $K(p=0,013)$; e entre a formulação elaborada com sucralose e a elaborada com acesulfame-K $(p=0,044)$; sendo que as formulações controle e sucralose, apresentaram os maiores valores, consideradas como muito boas. De acordo com os comentários dos julgadores, expressos na ficha de avaliação sensorial, a formulação com acesulfame-K e a formulação com a combinação de sucralose+acesulfame-K apresentaram um leve residual metálico.

Para o atributo acidez, a análise dos resultados evidenciou existir diferença significativa somente entre a formulação controle e a formulação elaborada com acesulfame-K $(p=0,024)$; sendo que a formulação controle foi considerada muito boa e a formulação com acesulfame-K apenas regular.

Aos 30 dias de armazenamento, a avaliação sensorial das compotas permitiu constatar que os atributos aparência, cor, brilho e maciez continuaram não apresentando diferença significativa entre as quatro formulações; neste período, para o atributo sabor, também não foi comprovada diferença significativa entre as formulações; porém, para o atributo acidez, foram observadas di- 
ferenças entre as formulações. Foi observada diferença significativa entre a formulação com acesulfame-K e a formulação com sucralose+acesulfame-K $(p=0,016)$; entre a formulação com acesulfame-K e a formulação com sucralose ( $p=0,023)$; e entre a formulação com sucralose+acesulfame-K e a formulação controle $(p=0,010)$. A formulação elaborada com acesulfame-K foi considerada inferior as demais; segundo comentários dos julgadores, devido a sua acidez pronunciada. Este fato pode ter influenciado o sabor geral desta compota, pois mesmo não tendo sido evidenciada diferença significativa para sabor, percebe-se na Figura 2, que esta formulação apresentou o menor valor médio para este atributo.

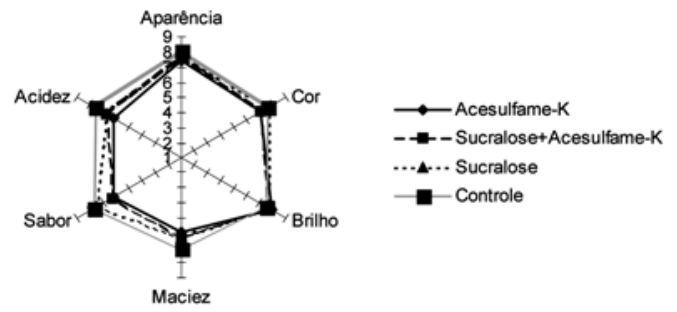

FIGURA 2 - Perfil de características das compotas de pêssego aos 30 dias de armazenamento

As pequenas variações que ocorreram aos 60 dias de armazenamento, não mostraram diferenças significativas entre as quatro formulações com relação aos atributos sensoriais de aparência, cor, brilho, maciez, sabor e acidez; porém, a formulação controle apresentou resultados ligeiramente mais altos que os outras em relação ao atributo sabor (Figura 3).

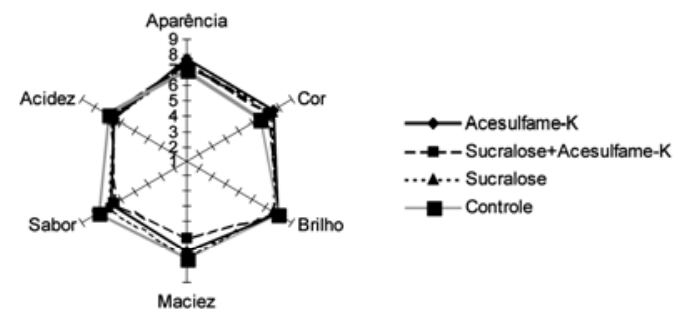

FIGURA 3 - Perfil de características das compotas de pêssego aos 60 dias de armazenamento

A avaliação sensorial das compotas do pêssego aos 90 dias de armazenamento (Figura 4) mostrou que os atributos de maciez e acidez não apresentaram diferenças significativas entre as formulações. Porém, foram comprovadas diferenças significativas entre as formulações em relação aos demais atributos. Diferenças significativas foram observadas entre a formulação com sucralose+acesulfame-K e a formulação com acesulfame-K, para os atributos aparência $(p=0,041)$, cor $(p=0,036)$ e brilho $(p=0,042)$; sendo que a formulação com acesulfame$\mathrm{K}$ apresentou os maiores valores. Diferenças significativas também foram verificadas entre a formulação com sucralose+acesulfame-K e a formulação controle em relação ao sabor $(\mathrm{p}=0,021)$. De acordo com os comentários dos julgadores, a formulação com sucralose+acesulfame- $\mathrm{K}$ foi considerada inferior à formulação de controle, devido ao residual amargo percebido nesta formulação.
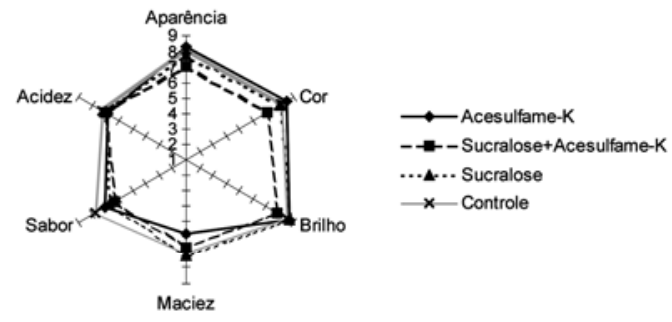

FIGURA 4 - Perfil de características das compotas de pêssego aos 90 dias de armazenamento

Baseado nos resultados obtidos para todas as formulações de compota de pêssego durante os 3 meses de armazenamento, pode-se verificar que as média de 7,2 a 8,3 para o atributo aparência; e 6,3 a 8,1 para o atributo cor, os qualificaram entre a descrições boa à ótima na escala de avaliação. Para o atributo brilho, os valores entre 7,2 e 8,6 os classificaram como brilhosos a extremamente brilhosos. Para o atributo maciez, os valores entre 5,6 a 7,4 classificaram as compotas na faixa entre moles e tenras. Os valores médios atribuídos para o atributo sabor, 5,4 a 7,7, qualificaram o sabor das compotas na faixa de bom a muito bom. Os valores médios conferidos ao atributo acidez, 5,4 a 7,6, caracterizaram a acidez das compotas do pêssego entre regular e muito boa.

De acordo com estes dados, verifica-se que o edulcorante sucralose apresenta-se com grande potencial para uso em compotas de pêssego, devido a sua similaridade com a compota controle, quanto aos atributos sensoriais, e também devido à excelente estabilidade mostrada durante o período de 90 dias de armazenamento.

O edulcorante acesulfame-K, apesar de conferir ligeira alteração de sabor, e acidez levemente pronunciada, também apresentou bons resultados quando avaliado sensorialmente; a combinação de acesulfame-K com sucralose pareceu minimizar a acidez; no entanto, na formulação elaborada com esta combinação, também foi percebido sabor ligeiramente estranho, lembrando metálico e amargo, próprios do edulcorante acesulfame-K [13, 40].

A Figura 5 mostra o resultado do teste de preferência das compotas de pêssego realizado aos 90 dias de armazenamento, com a participação de 100 consumidores de ambos os sexos, com idades entre 18 e 50 anos, pertencentes à comunidade universitária. Foi considerado para a soma de ordens o valor 1 para compota mais preferida, e o valor 4 para a menos preferida; desta forma, a compota que apresentou o menor valor de soma de ordens foi a mais preferida.

Analisando os resultados mostrados na Figura 5, verificou-se que não houve diferença significativa entre as 


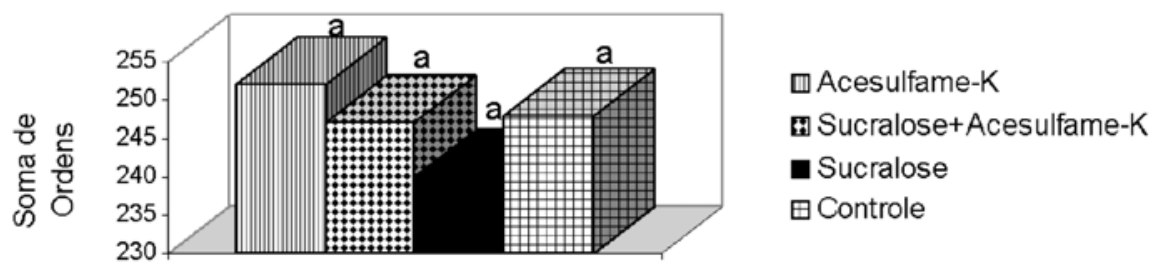

Formulações

FIGURA 5 - Preferência dos consumidores pelas compotas de pêssego

formulações em relação a preferência dos consumidores; desta forma, todas as formulações foram, estatisticamente, igualmente preferidas; os consumidores manifestaram, ainda, que havia grande semelhança entre as amostras sendo dificil ordená-las. Porém, pode-se observar pelo gráfico (Figura 5) uma pequena tendência de preferência pela formulação elaborada com sucralose, a qual obteve menor escore de pontos; a formulação de controle e a formulação com sucralose+acesulfame-K apresentaram valores semelhantes, e a formulação com acesulfame-K mostrou menor preferência, tendo recebido maior pontuação.

\section{4 - CONCLUSÕES}

- O uso individual do edulcorante sucralose proporcionou à compota de pêssego light características sensoriais similares à compota de pêssego convencional;

- a combinação de sucralose e acesulfame-K, na proporção de 1:1, proporcionou pequena vantagem sensorial à compota de pêssego, em relação ao uso isolado de acesulfame-K, quanto ao atributo acidez;

- as compotas de pêssego light permaneceram praticamente estáveis durante o período de 90 dias de armazenamento;

- $\quad$ as quatro formulações das compotas de pêssego não mostraram diferenças estatísticas quanto preferência dos consumidores, embora o melhor resultado tenha sido obtido pela compota elaborada com sucralose.

\section{5 - REFERÊNCIAS BIBLIOGRÁFICAS}

[1] ADA Reports. Position of the american dietetic association: use of nutritive and nonnutritive sweeteners. J. Am. Diet. Assoc., v. 104, p. 255-275, 2004

[2] BARNDT, R. L.; JACKSON, G. Stability of sucralose in baked goods. Food Technol., Chicago, v. 44, n. 1, p. 62-66, 1990.

[3] BARTOSHUK, L.M. Sweetness: history, preference, and genetic variability. Food Technol., v. 45, n. 11, p. 108113, 1991.
[4] BELL, J. High intensity sweeteners - a regulatory update. Food Technol., v. 47, p. 136, 1993.

[5] BORNSTEIN, B.L.; WIET, S.G.; POMBO, M. Sweetness adaptation of some carbohydrate and high potency sweeteners. J. Food Sci., v. 58, n. 3, p. 595-598, 1993.

[6] BRASIL. Ministério da Saúde. Secretaria de Vigilância Sanitária. Portaria no 32, de 17 de outubro de 1989. Aprova o uso de Acesulfame- $\mathrm{K}$ como aditivo para alimentos. Diário Oficial [da República Federativa do Brasil], Brasília, n 200,19 out. 1989.

[7] BRASIL. Ministério da Saúde. Secretaria de Vigilância Sanitária. Portaria no 318 de 24 de novembro de 1995. Aprova o uso de Sucralose com a função de edulcorante em alimentos e bebidas dietéticas. Diário Oficial [da República Federativa do Brasil], Brasília, n 229, 28 nov.

[8] CAMPOS, A.M.; CÂNDIDO, L.M.; RODRIGUEZ, R.M.H.P. Avaliação sensorial de molho de maçã com diferentes edulcorantes. Boletim CEPPA, v. 10, n. 2, p. 135-150, 1992.

[9] CÂNDIDO, L.M.B.; CAMPOS, A.M. Alimentos para fins especiais: Dietéticos. São Paulo: Ed. Varela, 1996. 411 p.

[10] CARDEllo, B.; DAMÁSIO, M. H. Edulcorantes e suas características: Revisão. Boletim SBCTA, v. 31, n. 2, p. 241-248, 1997.

[11] FOCUS ON... High intensity sweeteners. Food Ingredient Data Service, n. 4, p. 52-55, 1992.

[12] GELARDI, R.C. The multiple sweetener approach and new sweeteners on the horizon. Food Technol., v. 41, n. 1, p. 123-124, 1987.

[13] GIESE, J.H. Alternative sweeteners and bulking agents. Food Technol., v. 47, n. 1, p.113-126, 1993.

[14] GRICE, H.C.; GOLDSMITH, L.A. Sucralose An Overview of the toxicity. Food Chem. Toxicology, v. 38, Suppl 2, p.1-6, 2000.

[15] GROTZ, L.V.; ROBERT R. HENRY; JANET B. MCGILL; MELVIN J. PRINCE; HARRY SHAMOON; J. RICHARD TROUT; F. XAVIER PI-SUNYERJ. Lack of effect of sucralose on glucose homeostasis in subjects with type 2 diabetes Am. Diet. Assoc., v. 103, p. 1607-1612. 2003.

[16] INTERNATIONAL ORGANIZATION FOR STANDARDIZATION. ISO 5497: Sensory Analisys Methodology - Guidelines for the preparation of samples 
for which direct sensory analisys is not feasible. Genève, 1982.4p.

[17] I N T ER NAT I O N A ORGA N I Z A T I N F OR STANDARDIZATION. ISO 4121: Sensory Analisys Methodology - Evaluation of food products by methods using scales, Genève, 1987.

[18] I N T E R N A T I O N A L ORGANIZATIO N F OR STANDARDIZATION. ISO 8589: Sensory Analisys General guidance for the design of test rooms. Genève, 1988.9p.

[19] I N T ER NAT I O N A L ORGA NIZATIO N F OR STANDARDIZATION. ISO 8586-1: Sensory Analisys General guidance for the selection, training and monitoring of assessors, Genève, 1993.

[20] I N T ER N A T I O N L ORGANIZATIO N F OR STANDARDIZATION. ISO 13299: Sensory Analisys Methodology - General guidance for establishing a sensory profile, Genève, 1995.

[21] JENNER, M. R. Sweeteners: Discovery, molecular design, and chemoreception. New York: American Chemical Society, 1991, cap. 6: Sucralose - How to make sugar sweeter

[22] KING, B.M.; ARENTS, P.; DUINEVELD, C.A.A.. A comparison of aspartame and sucrose with respect to carryover efects in yogurt. Food Quality and Preference, v. 14, p. $7581,2003$.

[23] MASUdA, T.; UENO, Y.; KITABATAKE, N. High yield secretion of the sweet-tasting protein lysozyme from the yeast Pichia pastoris. Protein Expression and Purification, v. 39, p. 3542, 2005.

[24] MENEZES, S.; VIEGAS, E. Dietéticos em alta. Alimentos e Tecnologia, São Paulo, p. 22-26, 1990.

[25] MEHINAGIC, E.; ROYERA, G.te; BERTRAND, D.; SYMONEAUX, R.; LAURENS, F.; JOURJON, F. Relationship between sensory analysis, penetrometry and visible NIR spectroscopy of apples belonging to diferent cultivars. Food Quality and Preference, v. 14, p. $473484,2003$.

[26] MORALES, H. R. Evaluation de la análisis sensorial. In: SIMPOSIUM IBEROAMERICANO DE ANÁLISIS SENSORIAL, 2, 1999, Santa Fe. Resumos... Santa Fe, México: Universidad Iberoamericana, 1999.

[27] MORI, E.E.M. Métodos sensoriais e físicos para avaliação de alimentos e bebidas: princípios e aplicação. Campinas: ITAL, 1982, cap. 1: Noções gerais sobre qualidade.

[28] PARSEHIAN, S.D. Edulcorantes-tipos. 17 set. 1999. Online. Disponivel na Internet: http: / /www.perinat.org.ar/edul1.html.

[29] PENNY, C. Sweetness with calorie reduction. Food Ingred. Proces. Int., p.15-19, 1992.

[30] SARDESAI, V. M.; WALDSHAN, T.H. Natural and synthetic intense sweeteners. J. Nutr. Bioch., v. 2, p. 236-244, 1991.

[31] SASAKI, Y. F.; KAWAGUCHI, S.; KAMAYA, A.; OHSHITA M.; KABASAWA, K.; IWAMA, K.; TANIGUCHI, K.; TSUDA, $\mathrm{S}$. The comet assay with 8 mouse organs: results with 39 currently used food additives. Mutation Res., v. 519, p. $103119,2002$.

[32] SCHIFFMAN, S.S.; BOOTH, B.J.; LOSEE, M.L. PECORE, S.D.; WARWICK, Z.S. Bitterness of sweeteners as a function of concentration. Brain Res. Bull., v. 36, n. 5, p. 505-513, 1995.

[33] SPLENDA - O que você precisa saber. São Paulo: Johnson \& Johnson, 1998. 10p. Folheto.

[34] STATSOFT - Statistica. Tulsa: Statsoft, v. 1, 1991.935p.

[35] STONE, H.; SIDEL, J.L. Sensory Evaluation Practices. 2 ed. Florida: Academic Press, 1993. 338 p.

[36] SUNETT. O conceito. São Paulo: Hoechst, 1998, 11 p. Folheto.

[37] SUZUKI, H.; ONISHI, H.; HISAMATSU, S.; MASUDA, K; TAKAHASHI, Y; IWATA, M; MACHIDA, Y. Acetaminophen-containing chewable tablets with suppressed bitterness and improved oral feeling. Int. J. Pharm., v. 278, p. 5161, 2004.

[38] TORNOUT, V.; PELGROMS, J.; MEEREN, V.D. Sweetness evaluation of mixtures of fructose with saccharin, aspartame or acesulfame-K. J. Food Sci., v. 50, p. $469-472,1985$.

[39] VERDI, R.J.; HOOD, L.L. Advantages of alternative sweetener blends. Food Technol., v. 47, n. 6, p. 94-100, 1993.

[40] WIET, S.; BEYTS, P.K. Sensory caracteristics of sucralose and other higth intensity sweeteners. $\mathbf{J}$. Food Sci., v. 57, n. 4, p.1014-1019, 1992. 